Reprod. Nutr. Dévelop., 1985, 25 (1 A), 141-152.

\title{
Contribution à l'étude de l'embryogenèse de l'appareil génital d'Helix aspersa ; mise en évidence de la participation de deux types cellulaires à l'édification de la gonade.
}

\author{
Bernadette GRIFFOND, Jacqueline BRIDE
}

Laboratoire de Zoologie et Embryologie, U.A. CNRS n $n^{\circ} 040687$

Faculté des Sciences et des Techniques

Place Maréchal-Leclerc, 25030 Besançon Cedex, France.

Summary. Investigation of the embryonic development of the genital apparatus in Helix aspersa : evidence for the involvement of two cell types in gonadal development.

Cytological studies carried out by light and electron microscopy demonstrated that the genital apparatus of Helix aspersa was dualistic in origin : the proximal gonoduct was of ectodermal origin and differentiated from an evagination of the palleal cavity ; the gonad rudiment consisted of two voluminous germ cells surrounded by small mesodermal cells.

The segregation of the germ cells occurred early, i.e. during embryonic life, before torsion. The origin of these cells was unknown.

Our data do not agree with the concept of a germinal epithelium in which male and female cells differentiate from indeterminate stem cells.

\section{Introduction.}

Les recherches consacrées à l'organogenèse de l'appareil génital et à la différenciation des cellules germinales chez les Mollusques ont fait l'objet de mises au point périodiques (Revues de Martoja, 1964 ; Raven, 1966 ; Tardy, 1970 ; Nieuwkoop et Sutasurya, 1981). En dépit de nombreux travaux, le problème de la nature (ectodermique ou mésodermique) de la gonade demeure posé et, comme le soulignent Nieuwkoop et Sutasurya (1981), l'origine des cellules germinales est encore virtuellement inconnue. Si chez certains Pulmonés tels que Lymnaea stagnalis, Arion circumscriptus, Acroloxus lacustris... des cellules germinales sont reconnues dès la vie embryonnaire (Fraser, 1946 ; Luchtel, 1972 ; Brisson, 1973 ; Brisson et Besse, 1975 ; Brisson et Régondaud, 1977), chez d'autres comme Limax maximus la gonade semble composée de cellules-souches, toutes semblables, capables de donner naissance à l'une quelconque des catégories constitutives de l'ovotestis (Hoffmann, 1922). Selon Ancel (1903) et Guyard (1971), les escargots Helix pomatia et Helix aspersa appartiendraient à ce second type et c'est seulement après la naissance que les cellules germinales se différencieraient par modifications cytologiques des cellules-souches. 
Après avoir réalisé l'étude de la gonade et du tractus post-embryonnaires d'Helix aspersa (Griffond et Bride, 1981 ; Enée et Griffond, 1983), nous avons examiné des embryons de plus en plus jeunes afin d'apporter des précisions sur les deux questions fondamentales suivantes: 1) A partir de quels feuillets embryonnaires l'appareil génital se met-il en place ? 2) Les cellules germinales se différencient-elles à partir de cellules gonadiques pluripotentes ou existe-t-il une ségrégation précoce de la lignée germinale?

\section{Matériel et méthodes.}

Matériel. - Les pontes d'Helix aspersa élevés au laboratoire sont conservées à l'obscurité, à $20^{\circ} \mathrm{C}$, dans une atmosphère saturée en humidité. Dans ces conditions, la vie embryonnaire dure 10 à 12 jours. Pour cette étude, 6 pontes ont été utilisées et dans chaque ponte, 3 embryons ont été fixés tous les jours.

Techniques. - Les embryons sont extraits de leur capsule albumineuse; lorsqu'ils possèdent une coquille, celle-ci est enlevée délicatement à l'aide de pinces fines. Les embryons entiers sont fixés $2 \mathrm{~h}$ à $4^{\circ} \mathrm{C}$ dans le mélange à volumes égaux de glutaraldéhyde à $2 \%$, tampon cacodylate de sodium $0,1 \mathrm{M}$ et $\mathrm{NaCl}$ $0,17 \mathrm{M}$ ( $\mathrm{p} 0: 250 \mathrm{mOsM}$ ). Après $12 \mathrm{~h}$ de lavage à $4^{\circ} \mathrm{C}$ dans le tampon cacodylate, ils sont post-fixés dans une solution d'acide osmique à $2 \%$, tampon cacodylate $0,2 \mathrm{M}(1 \mathrm{vol} / 1 \mathrm{vol}, \mathrm{p} 0=240 \mathrm{mOsM})$ pendant $2 \mathrm{~h}$ à $20^{\circ} \mathrm{C}$. Les inclusions sont réalisées dans le mélange épon-araldite (Anderson et Ellis, 1965) dans lequel la proportion de DMP 30 est ramenée à 1,7\% (Mollenhauer, 1964). Les coupes semi-fines sont colorées au bleu de toluidine (Trump et al., 1961) et les ultrafines contrastées à l'acétate d'uranyle puis au citrate de plomb (Reynolds, 1963).

\section{Résultats.}

1) Etude du développement embryonnaire de l'appareil génital.

a) Différenciation de la gonade : La première ébauche gonadique est observée juste avant la torsion qui caractérise l'embryogenèse des Gastéropodes c'està-dire au $5^{\mathrm{e}}$ ou $6^{\mathrm{e}}$ jour de la vie embryonnaire chez Helix aspersa. Postérieure à la poche de résorption de l'albumen, elle est située dans une lacune hémocoelienne, en avant de l'intestin terminal, en arrière d'un amas de tissu nerveux qui correspond vraisemblablement au futur ganglion viscéral et à proximité d'une corne de la cavité palléale avec laquelle elle n'est cependant pas en continuité (fig. 1, PI. I).

Elle est constituée de 2 (exceptionnellement 1) cellules volumineuses (15 à $20 \mu \mathrm{m}$ de diamètre), arrondies ou ovales, reconnaissables à leur noyau clair renfermant des amas d'un matériel peu colorable au bleu de toluidine, qui semblent rayonner autour d'un gros nucléole central (fig. 2, PI. I). Ces 2 cellules qui représentent les gonocytes primordiaux ou cellules germinales primordiales sont associées à des cellules plus petites, allongées, dont les noyaux ont un aspect granu- 
leux. Peu nombreuses aux stades les plus précoces, de telles cellules, d'origine mésenchymateuse, s'incorporent à l'ébauche gonadique pendant toute la vie embryonnaire et même après la naissance (Griffond et Bride, 1981). L'ensemble cellules germinales et cellules accompagnatrices constitue un massif à bords irréguliers, qui en coupe transversale apparaît comme une cupule (souvent incomplète face à la poche de résorption de l'albumen) ou comme un anneau de petites cellules sombres entourant les cellules germinales primordiales plus claires (fig. 3, PI. I). Des mitoses sont fréquemment observées parmi les cellules non germinales mais l'aspect de la gonade se modifie peu ; durant les jours qui précèdent la naissance, les cellules germinales, toujours au nombre de 2, peuvent posséder 2 nucléoles (fig. 4, PI. I). C'est juste avant ou après l'éclosion que leur nombre est porté à 4 ou 6 .

b) Individualisation du gonoducte : Au moment où elle se met en place, la gonade est un massif isolé, sans relation avec les organes voisins. A partir du $6^{e}$ jour, I'épithélium de la cavité palléale, dans lequel se déroulent de nombreuses mitoses, émet une évagination en direction de l'ébauche gonadique; la fusion s'effectue vers 6 jours $1 / 2$ ou 7 jours (fig. 5 , PI. I). Au fur et à mesure du développement embryonnaire, cette évagination s'allonge et $s^{\prime}$ organise en un canal qui constitue la partie supérieure du tractus génital (fig. 6, PI. I).

Vers le $8^{\mathrm{e}}$ ou le $9^{\mathrm{e}}$ jour, une seconde évagination de la cavité palléale apparaît, non loin de la première (fig. $7, \mathrm{PI}$. 1). Le canal auquel elle donne naissance se dirige vers la région antérieure de l'embryon et sera à l'origine de la portion moyenne du tractus. Par la suite, les deux canaux situés dans le prolongement l'un de l'autre se séparent de la cavité palléale et, à la naissance, le conduit génital est un simple cordon filiforme (Enée et Griffond, 1983).

\section{2) Etude ultrastructurale des ébauches de l'appareil génital.}

a) La partie proximale du gonoducte, correspondant à la première évagination de la cavité palléale, est constituée de cellules épithéliales banales, à noyau basal riche en hétérochromatine (fig. 1, PI. II). Ces cellules sont solidement ancrées les unes aux autres dans leur région apicale par des desmosomes apicaux suivis de jonctions septées. Lorsque la jonction entre canal et gonade vient de se réaliser, des fragments de lame basale persistent au niveau de la zone d'accolement (fig. 2, PI. II) puis à partir du $8^{e}$ jour, aucun indice ne permet plus de suspecter qu'il y a eu fusion de deux ébauches distinctes.

b) Les cellules germinales : au niveau ultrastructural, les 2 cellules germinales d'une gonade embryonnaire ne montrent pas de différences importantes l'une par rapport à l'autre (fig. 3 et 4 , PI. II). Leur noyau clair renferme une chromatine finement dispersée et un gros nucléole central bien rond puis vers la fin de la vie embryonnaire souvent deux nucléoles. Dans leur cytoplasme riche en ribosomes libres et polyribosomes, l'ergastoplasme est peu abondant; les mitochondries allongées ont une matrice moyennement opaque aux électrons et des crêtes peu nombreuses ; l'appareil de Golgi est représenté par plusieurs dictyosomes locali- 
sés à proximité du noyau ; quelques gouttelettes lipidiques, corps multivésiculaires et lysosomes sont visibles. L'enveloppe nucléaire est percée d'une multitude de pores suggérant l'importance des échanges nucléo-cytoplasmiques (fig. 5, PI. II).

c) Les cellules accessoires : organisées en couronne autour des cellules germinales, elles sont polymorphes (fig. 1, PI. III). Leur noyau apparaît sombre par rapport à celui des cellules germinales primordiales; il renferme un nucléole à contour irrégulier, entouré de chromatine associée et une hétérochromatine assez abondante. Dans le cytoplasme, les mitochondries nombreuses possèdent de rares crêtes transversales; l'ergastoplasme forme de longues lames aplaties; deux centrioles sont souvent repérés ainsi qu'un ou deux dictyosomes, des corps multivésiculaires et des inclusions denses aux électrons (fig. 2, PI. III). Peu étendues aux stades précoces, des plages polysaccharidiques occupent progressivement une part importante des cellules accessoires. Zonula adherens et jonctions septées unissent ces cellules entre elles. A la périphérie de l'ébauche gonadique, la lame basale, mince, demeure incomplète durant toute la vie embryonnaire et des cellules présentant les caractères cytologiques des cellules du mésenchyme environnant (ergastoplasme abondant en lames dilatées) s'incorporent à la gonade

\section{PLANCHE I}

FIG. 1. - Coupe antéro-postérieure d'un embryon de 5 jours. Localisation de l'ébauche gonadique (petite flèche).

$\mathrm{B}$ : bouche; $\mathrm{Bb}$ : bulbe buccal ; $\mathrm{cp}$ : cavité palléale; gc : glande coquillière ; $\mathrm{P}$ : pied ; pa : grosses cellules de l'endoderme limitant la poche de résorption de l'albumen ; TD : intestin terminal ; grande flèche : ganglion viscéral.

$\times 75$

FIG. 2. - Embryon de 6 jours. La réalisation de coupes sériées montre que la gonade n'a pas de relation anatomique avec la cavité palléale. Autour d'une cellule germinale primordiale (flèche) à noyau clair et gros nucléole, les cellules mésenchymateuses ne sont pas encore organisées.

$\times 480$

FIG. 3. - Embryon de 6 jours. Les cellules accompagnatrices forment une cupule régulière (flèche) autour d'une cellule germinale primordiale.

pa : poche de résorption de l'albumen.

FIG. 4. - Embryon de 10 jours. Les cellules germinales (flèche) possèdent souvent 2 nucléoles à la fin de la vie embryonnaire.

FIG. 5. - Face à l'ébauche gonadique (petite flèche), la cavité palléale $d^{\prime}$ : $n$ embryon de 6 jours 1/2 émet une évagination (grande flèche). pa : poche de résorption de l'albumen.

FIG. 6. - Embryon de 7 jours. L'évagination de la cavité palléale (cp) s'organise en un canal. Les 2 cellules germinales $(\mathrm{cg})$ sont bien visibles. Certaines cellules endodermiques à noyau clair et gros nucléole (flèches) semblent prêtes à quitter la paroi de la poche de résorption de l'albumen (pa).

FIG. 7. - Vers le $8^{e}$ jour de la vie embryonnaire, une seconde évagination de la cavité palléale (flèche 2) apparaît dans le prolongement de la première (flèche 1).

c : cœur ; $\mathrm{cp}$ : cavité palléale ; pa : poche de résorption de l'albumen ; $r$ : rein ; TD : intestin. 
(fig. 3, PI. III) selon le processus que nous avons décrit après la naissance (Griffond et Bride, 1981).

d) Relations entre cellules germinales primordiales et cellules accessoires: alors que gonocytes et cellules accompagnatrices sont séparés par des espaces intercellulaires largement dilatés, leurs membranes sont, par endroits, étroitement juxtaposées, formant même des macula adherens (fig. 4, PI. III). Parfois également nous observons des figures suggérant l'existence de continuités cytoplasmiques entre cellules germinales et accessoires, il ne s'agit pas de véritables ponts mais de zones étroites au niveau desquelles nous ne distinguons pas de séparation membranaire (fig. 5, PI. III).

\section{Discussion.}

1) Origine de l'appareil génital : chez Helix aspersa, les voies génitales et la gonade se forment de façon indépendante, à partir d'ébauches distinctes qui fusionnent secondairement. A la différence des autres Stylommatophores (Hoffmann, 1922 ; Laviolette, 1954 ; Luchtel, 1972), l'organogenèse génitale de l'escargot se rattache donc à la théorie dualiste dont Eisig (1869) fut le premier à poser les principes. Sur ce point, nous confirmons les conclusions d'Ancel (1903) chez Helix pomatia et Buresch (1912) chez Helix arbustorum. Le gonoducte a une origine ectodermique : ses régions supérieure et moyenne, que nous avons plus spécialement étudiées, se forment à partir de deux évaginations de la cavité palléale. La gonade, quant à elle, renferme des composants mésodermiques, petites cellules du mésenchyme qui durant toute la vie embryonnaire et même après la naissance paraissent attirées par les deux cellules germinales primordiales autour desquelles elles viennent s'organiser. En ce qui concerne ces cellules germinales, leur origine demeure énigmatique : en effet chez les embryons très jeunes, ou bien nous ne les repérons pas, ou bien elles sont en place et aucun indice alors ne nous permet de savoir d'où elles proviennent. Chez les autres Mollusques (Basommatophores essentiellement) où des cellules comparables ont été décrites,

\section{PLANCHE II}

FIG. 1. - Chez un embryon de 7 jours, la jonction est effectuée entre l'évagination de la cavité palléale (Ev) et l'ébauche gonadique (Go) dont les 2 cellules germinales primordiales (étoiles) sont visibles. Dans l'épithélium de la cavité palléale (cp), les mitoses (flèches) sont nombreuses.

Tm : tissu mésenchymateux.

$\times 2350$

FIG. 2. - Détail de la zone de jonction, encadré sur la figure 1: une lame basale (flèches) persiste encore.

FIG. 3 et 4 . - U/trastructure des 2 cellules germinales primordiales.

$\mathrm{G}$ : appareil de Golgi, ly : Iysosomes, $\mathrm{m}$ : mitochondrie, $\mathrm{N}:$ noyau, $\mathrm{n}:$ nucléole.

$\times 7800$

FIG. 5. - Les pores nucléaires (flèches) sont particulièrement abondants dans les cellules germinales primordiales. 
elles sont généralement considérées comme ayant une origine mésodermique (Fraser, 1946 ; Brisson et Régondaud, 1971 et 1977). Luchtel (1972), partisan de la théorie uniciste, attribue aux cellules germinales de la limace une origine ectodermique mais ne rejette pas la possibilité que des cellules mésodermiques ou endodermiques puissent migrer et coloniser l'ébauche ectodermique. Chez $\mathrm{Helix}$ aspersa, nous n'avons aucun argument en faveur d'une origine ectodermique des cellules germinales ; par contre, nous pensons qu'elles peuvent être mésodermiques comme le suggère leur localisation au sein du compartiment mésenchymateux ou même endodermiques, provenant de la paroi de la poche de résorption de l'albumen proche de l'ébauche gonadique, dont des cellules à noyau clair et gros nucléole semblent parfois prêtes à se détacher (fig. 6, PI. I) ; rappelons à ce sujet que, chez le Xénope, les cellules germinales primordiales sont d'abord localisées dans l'endoderme (Kalt, 1973).

2) Présence de deux lignées cellulaires dans la gonade: au cours de l'embryogenèse gonadique d'Helix aspersa, nous n'avons jamais observé l'existence d'un stade à un seul type cellulaire indifférencié ; aux stades les plus précoces, dès que nous pouvons reconnaître l'ébauche gonadique, elle est constituée de deux catégories de cellules: les gonocytes primordiaux dont les caractères ultrastructuraux sont semblables à ceux décrits dans de nombreuses espèces animales (Bruslé, 1971, 1972) et des cellules non germinales disposées à leur périphérie. Nos observations rejoignent celles réalisées chez les Basommatophores (Fraser, 1945 ; Brisson et Besse, 1975 ; Brisson et Régondaud, 1977) mais sont en contradiction avec celles d'Ancel (1903) et Guyard (1971) qui décrivent la gonade d'escargots à la naissance comme constituée d'un seul type de cellules indifférenciées pluripotentes, souches de toutes les cellules germinales et accessoires de l'ovotestis. En réalité, nos travaux démontrent que la ségrégation de la

\section{PLANCHE III}

FIG. 1. - Coupe transversale de la gonade d'un embryon de 6 jours. Les cellules accompagnatrices (ca) forment un anneau autour d'une cellule germinale primordiale $(\mathrm{cg})$.

$\mathrm{n}$ : nucléole, Tm : tissu mésenchymateux.

FIG. 2. - Détail des cellules accompagnatrices.

$\mathrm{cd}$ : corps denses, $\mathrm{cm}$ : corps multivésiculaire, er : lame ergastoplasmique, $\mathrm{G}$ : appareil de Golgi, $\mathrm{m}$ : mitochondrie, $\mathrm{N}$ : noyau.

FIG. 3. - Dans un embryon de 8 jours, une cellule du mésenchyme (me) s'accole au massif gonadique $(\mathrm{Go})$ le long duquel une lame basale ténue et discontinue est visible (flèche). mu : fibre musculaire.

$\times 15600$

FIG. 4. - Embryon de 6 jours. Cellules germinales primordiales (cg) et cellules accompagnatrices (ca) sont séparées par un large espace intercellulaire qui se resserre localement au niveau de macula adherens par exemple (flèche).

FIG. 5. - Elles peuvent aussi émettre des prolongements qui s'intriquent étroitement ; parfois les limites cellulaires imprécises suggèrent l'existence de continuité cytoplasmique entre les deux types cellulaires (flèche).

$\mathrm{cg}$ : cellule germinale primordiale, ca : cellule accompagnatrice. 
lignée germinale est relativement précoce chez Helix aspersa puisque nous repérons les initiales germinales dans la première moitié de la vie embryonnaire. II serait intéressant de pouvoir les caractériser plus tôt afin de savoir si, comme le suggérait Woods $(1931,1932)$ chez un lamellibranche, les cellules germinales sont les descendantes directes du micromère $4 \mathrm{~d}$ ou si, comme le pensent Nieuwkoop et Sutasurya (1981) et Nieuwkoop (1982), les mollusques représentent un exemple de mécanisme intermédiaire entre la " préformation » et la détermination épigénétique des gonocytes.

3) Devenir des cellules germinales : pendant la vie embryonnaire, les cellules germinales primordiales ne se divisent pas, elles sont en repos cinétique. Des constatations identiques ont été faites dans d'autres groupes animaux (Gipouloux, 1975 ; Brisson et Régondaud, 1977). Les premières multiplications de ces cellules se produisent au moment de la naissance. ( $N$ 'ayant considéré la gonade qu'après l'éclosion, Guyard (1971) pouvait difficilement suspecter l'existence des cellules germinales primordiales car leurs cellules-filles ont des dimensions plus réduites et sont moins facilement discernables). Dès lors, à toutes les étapes de la vie (croissance, phases du cycle sexuel, hibernation prolongée...) des cellules germinales sont présentes dans l'épithélium qui tapisse les acini. Le fait que jamais nous n'observions des étapes de transition entre cellules somatiques et cellules germinales nous incite à considérer les deux cellules germinales primordiales comme étant à l'origine de toutes les cellules germinales de l'ovotestis, selon des modalités que nous tentons actuellement de préciser. La notion d'épithélium germinatif renfermant des cellules-souches pluripotentes capables de se différencier, selon les conditions, en gamètes mâles ou femelles ou en cellules accessoires nourricières ou folliculaires ne nous semble pas rendre compte de la réalité et comme Luchtel (1972) nous pensons que l'épithélium des acini est en fait un épithélium banal au sein duquel sont disséminées des cellules germinales.

La sexualisation des cellules germinales est un problème particulièrement complexe chez les animaux hermaphrodites : les deux gonocytes primordiaux de l'escargot sont-ils rigoureusement identiques, donnant naissance à des générations de cellules germinales qui évolueront dans le sens mâle ou femelle selon leur microenvironnement ou bien sont-ils programmés différemment, I'un étant la cellule-mère de la lignée mâle, l'autre celle de la lignée femelle ? Au niveau ultrastructural, nous n'avons pas observé de différences entre eux mais les nombreuses questions soulevées mettent en évidence la nécessité de trouver des marqueurs spécifiques de la lignée germinale ou de la lignée somatique d'une part, de la lignée mâle ou de la lignée femelle d'autre part.

\section{Conclusion.}

Chez Helix aspersa, l'appareil génital se met en place selon le mode défini par la théorie dualiste. Le tractus a une origine ectodermique tandis que la gonade est, au moins partiellement, d'origine mésodermique. 
Contrairement aux données antérieures, il apparaît que la lignée germinale est déterminée de façon précoce mais l'origine des cellules germinales primordiales de même que leur devenir ne sont pas totalement élucidés. Nos observations remettent en cause la notion d'épithélium germinatif composé de cellules pluripotentes capables de donner indifféremment des cellules germinales ou des cellules somatiques.

En définitive, l'ovotestis d'Helix aspersa constitue un exemple passionnant de différenciation mettant en jeu conjointement des interactions cellulaires et l'influence de l'environnement hormonal.

Reçu en juillet 1984.

Accepté en octobre 1984.

\section{Références}

ANCEL P., 1903. Histogenèse et structure de la glande hermaphrodite d'Helix pomatia L. Th. Doct. Sci. nat., Univ. Nancy, $n^{\circ} 16$.

ANDERSON W. A., ELLIS R. A., 1965. Ultrastructure of Trypanosoma lewisi : flagellum, microtubules and the kinetoplast. J. Protozool., 12, 483-499.

BRISSON P., 1973. Observation ultrastructurale des cellules germinales chez l'embryon d'Acroloxus lacustris (L.) (Gastéropode Pulmoné Basommatophore). C. R. Acad. Sci. Paris, 277, 2205-2208.

BRISSON P., BESSE C., 1975. Etude ultrastructurale de l'ébauche gonadique chez l'embryon de Lymnaea stagnalis L. (Gastéropode Pulmoné Basommatophore). Bull. Soc. Zool. Fr., 100. 345-349.

BRISSON P., RÉGONDAUD J., 1971. Observations relatives à l'origine dualiste de l'appareil génital chez quelques Gastéropodes Pulmonés Basommatophores. C. R. Acad. Sci. Paris, 273, 2339 2341.

BRISSON P., RÉGONDAUD J., 1977. Origine et structure de l'ébauche de la gonade chez les Gastéropodes Pulmonés Basommatophores. Malacologia, 16, 457-466.

BRUSLÉ J., 1971. Les infrastructures germinales mâles précoces (gonocytes, spermatogonies et spermatocytes I). Ann. Biol., 10, 353-402.

BRUSLE J., 1972. Les infrastructures germinales femelles précoces (gonocytes, ovogonies et ovocytes I). Ann. Biol., 11, 505-571.

BURESCH I., 1912. Untersuchungen über die Zwitterdrüse der Pulmonaten. I. Die Differenzierung der Keimzellen bei Helix arbustorum. Arch. Zellforsch., 7, 314-343.

EISIG H., 1869. Beiträge zur Anatomie und Entwicklungsgeschichte der Geschlechts-organe von Limnaeus. Zeit. Wiss. Zool., 19, 297-320.

ENEE J., GRIFFOND B., 1983. Etude histologique et ultrastructurale de l'organogenèse et de la différenciation post-embryonnaires de l'appareil génital d'Helix aspersa Müller, Reprod. Nutr. Dévelop., 23, 927-941.

FRASER L. A., 1946. The embryology of the reproductive tract of Lymnaea stagnalis appressa Say. Trans. amer. Micr. Soc., 65, 279-298.

GIPOULOUX J. D., 1975. "Cytoplasme germinal " et détermination germinale chez les Amphibiens Anoures. Ann. Biol., 14, 476-487.

GRIFFOND B., BRIDE J., 1981. Etude histologique et ultrastructurale de la gonade d'Helix aspersa Müller à l'éclosion. Reprod. Nutr. Dévelop., 21, 149-161.

GUYARD A., 1971. Etude de la différenciation de l'ovotestis et des facteurs contrôlant l'orientation sexuelle des gonocytes de l'Escargot Helix aspersa Müller. Th. Doct. Sci. nat., Univ. Besançon, $n^{\circ} 56$.

HOFFMANN H., 1922. Ueber die Entwicklung der Geschlechtorgane bei Limax maximus. Zeit. Wiss. Zool., 119, 493-536. 
KALT M. R., 1973. Ultrastructural observations on the germ line of Xenopus laevis. Z. Zelfforsch., 138, 41-62.

LAVIOLETTE P., 1954. Etude cytologique et expérimentale de la régénération germinale après castration chez Arion rufus L. (Gastéropode pulmoné). Ann. Sci. nat. Zool. Biol. anim., 16, 427-530.

LUCHTEL D., 1972. Gonadal development and sex determination in Pulmonate Molluscs. I. Arion circumscriptus. Z. Zellforsch., 130, 279-301.

MARTOJA M., 1964. Développement de I'appareil reproducteur chez les Gastéropodes Pulmonés. Ann. Biol., 3, 199-232.

MOLLENHAUER H. H., 1964. Plastic embedding mixtures for use in electron microscopy. Stain Technol., 39, 111-114.

NIEUWKOOP P. D., 1982. La détermination et la migration des cellules germinales dans le règne animal. Ann. Biol., 21, 37-51.

NIEUWKOOP P., SUTASURYA L. A., 1981. Primordial germ cells in the invertebrates. Cambridge University Press.

RAVEN C. P., 1966. Morphogenesis, the analysis of Molluscan development. 2nd ed., Pergamon Press, Oxford.

REYNOLDS E. S., 1963. The use of lead citrate at high $\mathrm{pH}$ as an electron opaque stain in electron microscopy. J. Cell. Biol., 17, 208-212.

TARDY M. J., 1970. Organogenèse de l'appareil génital chez les Moliusques. Bull. Soc. Zool. Fr., 95, 407-428.

TRUMP B. F., SMUCKLER E. A., BENDITT E. P., 1961. A method for staining epoxy sections for light microscopy. J. Ultrastruct. Res., 5, 343-345.

WOODS F. H., 1931. History of the germ cells in Sphaerium striatinum (Lam.). J. Morphol. Physiol., 51, 545-595.

WOODS F. H., 1932. Keimbahn determinants and continuity of the germ cells in Sphaerium striatinum (Lam.). J. Morphol., 53, 345-365. 